\title{
GENERALIZED TEMPERLEY-LIEB ALGEBRAS AND DECORATED TANGLES
}

\author{
R.M. GREEN \\ Department of Mathematics and Statistics \\ Lancaster University \\ Lancaster LA1 4YF \\ England \\ E-mail: r.m.green@lancs.ac.uk
}

\begin{abstract}
We give presentations, by means of diagrammatic generators and relations, of the analogues of the Temperley-Lieb algebras associated as Hecke algebra quotients to Coxeter graphs of type $B$ and $D$. This generalizes Kauffman's diagram calculus for the Temperley-Lieb algebra.
\end{abstract}

\section{To appear in the Journal of Knot Theory and its Ramifications}

\section{INTRODUCTION}

The Temperley-Lieb algebra is a certain finite dimensional associative algebra which first arose in [14] in the context of Potts models in statistical mechanics. As well as having applications to physics, the algebra also appears in the framework of knot theory, where it is closely related to the Jones polynomial and isotopy invariants of links. This relationship is explained in [10], where it is shown that the Temperley-Lieb algebra occurs naturally as a quotient of the Hecke algebra arising from a Coxeter system of type $A$.

In his thesis, Graham [6] generalized this realization of the Temperley-Lieb algebra as a Hecke algebra quotient to the case of a Coxeter system of arbitrary type. These Hecke algebra quotients are the eponymous "generalized TemperleyLieb algebras". Graham classified the finite dimensional generalized TemperleyLieb algebras into seven infinite families: $A, B, D, E, F, H$ and $I$, where the family of type $A$ gives the original Temperley-Lieb algebras.

In this paper, we give presentations, by means of diagrammatic generators and relations, of the generalized Temperley-Lieb algebras of types $B$ and $D$, building on the work of tom Dieck [2] and that of Martin and Saleur [13]. The motivation behind this is that Kauffman's pictorial formation of the type $A$ algebras [11] 
has been of great value when it comes to understanding otherwise purely abstract algebraic computations such as the representation theory and cellular structures (in the sense of $[\mathbf{7}])$. The algebra of type $A$ is also of great value in knot theory $[\mathbf{1 0}]$, and is a central object in the theory of quantum groups. This is because the type $A$ Artin group is both the "standard" topological braid group, and a deformation of the symmetric group. It is possible, although not yet clear, that the generalized braid groups may be of some importance in these areas.

\section{Generalized Temperley-Lieb algebras of types $B$ and $D$}

Our main objects of study are the generalized Temperley-Lieb algebras arising from Coxeter systems of types $B$ and $D$. These may readily be described in terms of generators and relations, as we now show.

The information required to define the algebras is encoded in the relevant Dynkin diagrams. The Dynkin diagrams of types $B_{n}$ and $D_{n}$ are numbered as in Figures 1 and 2 respectively.

Figure 1. Dynkin diagram of type $B_{n}$

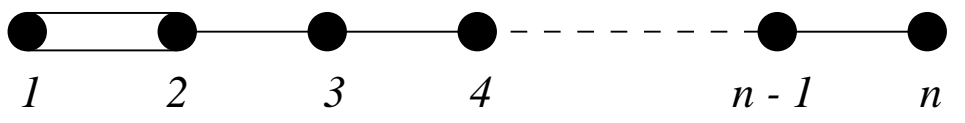

Figure 2. Dynkin diagram of type $D_{n}$

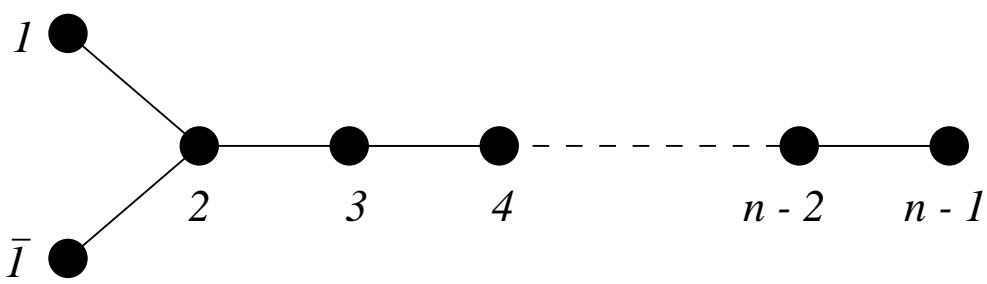

We generate the Hecke algebra (as in $[\mathbf{9}, \S 7]$ ) by its Kazhdan-Lusztig basis elements

$$
B_{s}=C_{s}^{\prime}:=v^{-1} T_{1}+v^{-1} T_{s}
$$

where $v$ is an indeterminate satisfying $v^{2}=q$ and $s$ is a generating involution of the Coxeter group.

Lemma 1.1. The Hecke algebra $\mathcal{H}\left(B_{n}\right)$ is generated as an algebra with identity by the set $\left\{B_{1}, B_{2}, \ldots, B_{n}\right\}$ and defining relations

$$
\begin{aligned}
B_{s}^{2} & =\left(v+v^{-1}\right) B_{s}, \\
B_{s} B_{t} & =B_{t} B_{s} \quad \text { if }|s-t|>1, \\
B_{s} B_{t} B_{s}-B_{s} & =B_{t} B_{s} B_{t}-B_{t} \quad \text { if }|s-t|=1 \text { and }\{s, t\} \neq\{1,2\}, \\
B_{s} B_{t} B_{s} B_{t}-2 B_{s} B_{t} & =B_{t} B_{s} B_{t} B_{s}-2 B_{t} B_{s} \quad \text { if }\{s, t\}=\{1,2\} .
\end{aligned}
$$

Lemma 1.2. The Hecke algebra $\mathcal{H}\left(D_{n}\right)$ is generated as an algebra with identity by the set $\left\{B_{\overline{1}}, B_{1}, B_{2}, \ldots, B_{n-1}\right\}$ and defining relations

$$
\begin{aligned}
B_{s}^{2} & =\left(v+v^{-1}\right) B_{s} \\
B_{s} B_{t} & =B_{t} B_{s} \quad \text { if } s \text { and } t \text { are not connected in the Dynkin diagram, }(2) \\
B_{s} B_{t} B_{s}-B_{s} & =B_{t} B_{s} B_{t}-B_{t} \quad \text { otherwise. }
\end{aligned}
$$


Proofs of Lemmas 1.1 and 1.2. A routine calculation shows that these definitions are equivalent to the usual ones involving the $T_{s}$ elements.

The generalized Temperley-Lieb algebra $T L(X)$ associated to a Coxeter system with graph $X$ is the quotient of the Hecke algebra $\mathcal{H}(X)$ obtained by factoring out the ideal $I(X)$ generated by the elements

$$
\sum_{w \in\left\langle s_{i}, s_{j}\right\rangle} T_{w}
$$

as the pairs $\left(s_{i}, s_{j}\right)$ run over pairs of adjacent nodes in the Dynkin diagram.

We denote the image in $T L(X)$ of $B_{s} \in \mathcal{H}(X)$ by $E_{s}$.

This construction generalizes the construction of the Temperley-Lieb algebra from the Hecke algebra of type $A$ (see [10]), and is due to Graham [6].

It is now not hard to describe the algebras $T L\left(B_{n}\right)$ and $T L\left(D_{n}\right)$ explicitly.

Proposition 1.3. Let $n \in \mathbb{N} \geq 2$. We define the associative, unital algebra $T L\left(B_{n}\right)$ over the ring $\mathcal{A}=\mathbb{Z}\left[v, v^{-1}\right]$ via generators $E_{1}, E_{2}, \ldots E_{n}$ and relations

$$
\begin{aligned}
E_{i}^{2} & =[2] E_{i}, \\
E_{i} E_{j} & =E_{j} E_{i} \quad \text { if }|i-j|>1, \\
E_{i} E_{j} E_{i} & =E_{i} \quad \text { if }|i-j|=1 \quad \text { and } i, j>1, \\
E_{i} E_{j} E_{i} E_{j} & =2 E_{i} E_{j} \quad \text { if }\{i, j\}=\{1,2\} .
\end{aligned}
$$

Here, $[2]:=v+v^{-1}$.

Proof. This follows from Lemma 1.1 once it has been observed that the extra relations imposed on $\mathcal{H}\left(B_{n}\right)$ to make $T L\left(B_{n}\right)$ are precisely those which set each side of equations (3) and (4) of Lemma 1.1 to zero.

We can apply the same arguments to type $D_{n}$, as follows.

Proposition 1.4. Let $n \in \mathbb{N} \geq 4$. We define the associative, unital algebra $T L\left(D_{n}\right)$ over the ring $\mathcal{A}=\mathbb{Z}\left[v, v^{-1}\right]$ via generators $E_{1}, E_{\overline{1}}, E_{2}, \ldots E_{n-1}$ and relations

$$
\begin{aligned}
E_{i}^{2} & =[2] E_{i}, \\
E_{i} E_{j} & =E_{j} E_{i} \quad \text { if } i \text { and } j \text { are not connected in the graph, } \\
E_{i} E_{j} E_{i} & =E_{i} \quad \text { if } i \text { and } j \text { are connected in the graph. }
\end{aligned}
$$

Proof. This follows from Lemma 1.2 along the lines of the proof of Proposition 1.3.

\section{DeCORATED TANGLES}

A convenient way to introduce the diagram calculi relevant to this paper is by means of the category of "decorated tangles". As well as being an important tool in this paper, the decorated tangles go on to play a key rôle in the sequel to this paper [8], which analyses the structure of the generalized Temperley-Lieb algebras of type $H$. 
We introduce a category, the morphisms of which are certain tangles. Our set-up tends to follow that of Freyd and Yetter [5], who showed how Kauffman's tangletheoretic approach to the Temperley-Lieb algebra may be defined in terms of certain categories.

A tangle is a portion of a knot diagram contained in a rectangle. The tangle is incident with the boundary of the rectangle only on the north and south faces, where it intersects transversely. The intersections in the north (respectively, south) face are numbered consecutively starting with node number 1 at the western (i.e. the leftmost) end.

Two tangles are equal if there exists an isotopy of the plane carrying one to the other such that the corresponding faces of the rectangle are preserved setwise.

We call the edges of the rectangular frame "faces" to avoid confusion with the "edges" which are the arcs of the tangle.

For our purposes, it is necessary to extend the notion of a tangle so that each arc of the tangle may be assigned a nonnegative integer. (This is similar to the notion of "coloured" tangles in [5].) If an arc is assigned the value $r$, we represent this pictorially by decorating the arc with $r$ blobs.

A decorated tangle is a crossing-free tangle in which each arc is assigned a nonnegative integer. Any arc not exposed to the west face of the rectangular frame must be assigned the integer 0 . This means that any decorated tangle consists only of loops and edges, none of which intersect each other.

Example. Figure 3 shows a typical example of a decorated tangle. We will tend to emphasise the intersections of the tangle with the frame rather than the frame itself, which is why each node (i.e. intersection point with the frame) is denoted by a disc.

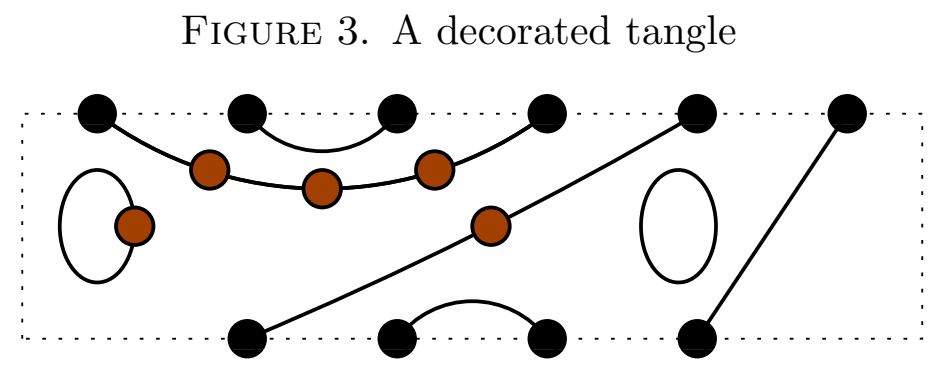

The category of decorated tangles, $\mathbb{D} \mathbb{T}$, has as its objects the natural numbers. The morphisms from $n$ to $m$ are the decorated tangles with $n$ nodes in the north face and $m$ in the south. The source of a morphism is the number of points in the north face of the bounding rectangle, and the target is the number of points in the south face. Composition of morphisms works by concatenation of the tangles, matching the relevant south and north faces together.

Note that for there to be any morphisms from $n$ to $m$, it is necessary that $n+m$ be even. Also notice that the asymmetric properties of the west face of the rectangle mean that we cannot introduce the tensor product of two morphisms by the lateral juxtaposition of diagrams as in [5].

These category-theoretic definitions allow us to define an algebra of decorated tangles. Let $R$ be a commutative ring and let $n$ be a positive integer. Then the $R$-algebra $\mathbb{D} \mathbb{T}_{n}$ has as a free $R$-basis the morphisms from $n$ to $n$, where the multiplication is given by the composition in $\mathbb{D} \mathbb{T}$.

The edges in a tangle $T$ which connect nodes (i.e. not the loops) may be classified into two kinds: propagating edges, which link a node in the north face with a node 
in the south face, and non-propagating edges, which link two nodes in the north face or two nodes in the south face.

It is convenient to define certain named tangles, $e_{\overline{1}}, e_{1}, e_{2}, \ldots, e_{n-1}$ and $e$, in the algebra $\mathbb{D T}_{n}$.

The tangle $e_{i}$ is defined as follows. There is an edge connecting nodes $i$ and $i+1$ in the north face to each other, and the same for the south face. For other nodes $k \neq i, i+1$, node $k$ in the north face is connected to node $k$ in the south face. There are no decorated edges and no loops.

The tangle $e_{\overline{1}}$ is obtained from $e_{1}$ by adding decorations to the two non-propagating edges.

The tangle $e$ has no loops and all its edges are propagating. There is one decorated edge, namely the one joining node 1 in the north face to node 1 in the south face.

Example. In the case $n=6$, the tangles $e, e_{2}$ and $e_{\overline{1}}$ are as shown in Figures 4,5 and 6 respectively.

Figure 4. The tangle $e$ in $\mathbb{D T}_{6}$

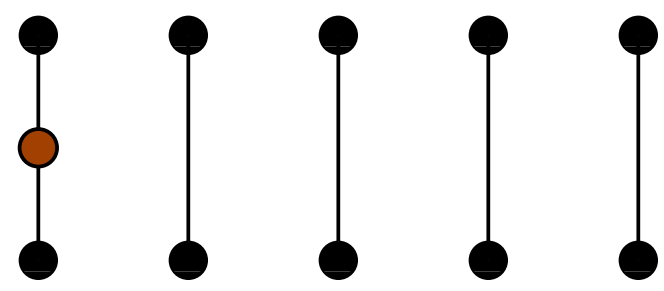

Figure 5. The tangle $e_{2}$ in $\mathbb{D}_{6}$
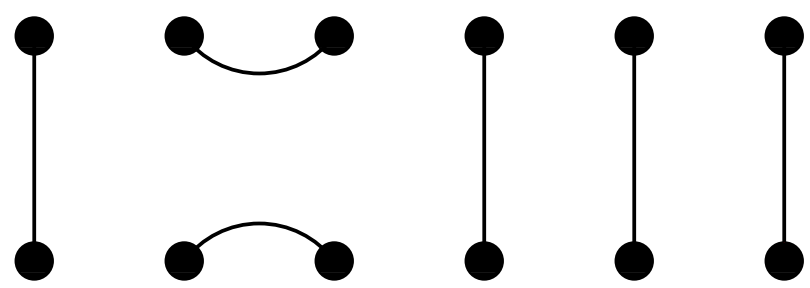

Figure 6 . The tangle $e_{\overline{1}}$ in $\mathbb{D T}_{6}$
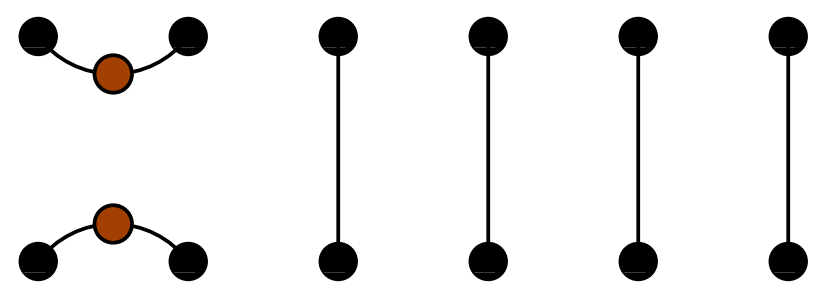

Note that if $e_{i}$ and $e_{j}$ are such that $i, j \neq \overline{1}$, then the relations $e_{i} e_{j}=e_{j} e_{i}$ (if $|i-j|>1$ ) and $e_{i} e_{j} e_{i}=e_{i}\left(\right.$ if $|i-j|=1$ ) hold in $\mathbb{D T}_{n}$.

\section{Review of Results in type $A$}

We now recall Kauffman's tangle-theoretic approach to the Temperley-Lieb algebra $T L_{n}$ in terms of the algebra $\mathbb{D T}_{n}$ of decorated tangles. Proofs may be found in $[\mathbf{1 2}]$.

Theorem 3.1 (Kauffman). Let $\delta$ be an indeterminate. Consider the subalgebra of $\mathbb{D T}_{n}[\delta]$ generated by the elements $e_{1}, e_{2}, \ldots, e_{n-1}$ (but not $e_{\overline{1}}$ ). Let $T L_{n}$ be the quotient of this subalgebra by the relation 


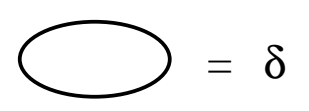

The algebra $T L_{n}$ is the Temperley-Lieb algebra, which has as a basis the set of all undecorated elements of $\mathbb{D T}_{n}$ with no loops. It is given by generators $e_{1}, e_{2}, \ldots e_{n-1}$ and defining relations

$$
\begin{aligned}
e_{i}^{2} & =\delta e_{i}, \\
e_{i} e_{i \pm 1} e_{i} & =e_{i}, \\
e_{i} e_{j} & =e_{j} e_{i} \quad \text { if }|i-j|>1 .
\end{aligned}
$$

What the relation involving the loop means is that each occurrence of an undecorated loop is removed, and the resulting tangle element is multiplied by the indeterminate $\delta$ to compensate.

It should be noted that no decorated edges or loops can arise, since the generators $e$ and $e_{\overline{1}}$ are not involved.

The relation $e_{1}^{2}=\delta e_{1}$ is illustrated in Figure 7 .

FiguRE 7. The relation $e_{1}^{2}=\delta e_{1}$ in $T L_{3}$

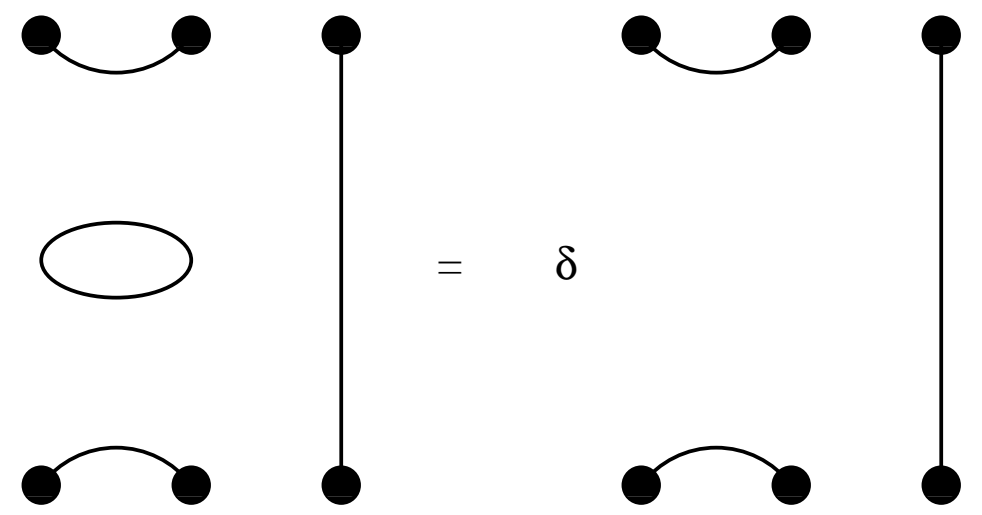

The rank of $T L_{n}$ is well-known to be equal to the Catalan number

$$
C(n):=\frac{1}{n+1}\left(\begin{array}{c}
2 n \\
n
\end{array}\right) \text {. }
$$

A basis may be described in terms of "reduced words" in the algebra generators $e_{i}$. A reduced word for $T L_{n}$ is a monomial in the generators $\left\{e_{1}, e_{2}, \ldots, e_{n-1}\right\}$ of minimal length. In other words, any sequence of relations in Theorem 3.1 which can be applied to the monomial consists only of applications of commutation relations.

It is clear that any word in the generators is of the form $\delta^{a}$ times a reduced word for some integer $a$, simply by repeated application of the relations. Thus the reduced words form a spanning set for the algebra. In fact, it is well-known that after discarding repeats, the reduced words give the same basis of $T L_{n}$ as the diagrams in Theorem 3.1.

Reduced words for $T L_{n}$ also have the following important property.

Lemma 3.2. Let $e_{w}=e_{i_{1}} e_{i_{2}} \cdots e_{i_{r}}$ be a reduced word in $T L_{n}$. Define $a:=$ $\min \left\{i_{1}, i_{2}, \ldots, i_{r}\right\}$ and $b:=\max \left\{i_{1}, i_{2}, \ldots, i_{r}\right\}$. Then there is exactly one occurrence of $e_{a}$ in $e_{w}$, and there is exactly one occurrence of $e_{b}$ in $e_{w}$.

Proof. This is a special case of [4, Lemma 4.3.5].

Another important property of $T L_{n}$ is that the number of occurrences of $e_{i}$ in a reduced word may be found by inspection of the corresponding diagram. 
Lemma 3.3. Let $D$ be a basis diagram for the Temperley-Lieb algebra $T L_{n}$. Assume the rectangular frame is drawn with nodes in positions $\{0,1\} \times\{1,2, \ldots, n\}$. Assume the diagram $D$ is drawn so that the total number, $2 \ell(D)$, of intersections of the associated link with the set of lines $x=k+1 / 2$ (as $k$ runs from 1 to $n-1$ ) is minimal.

Let $e_{w}$ be a reduced monomial in the generators $\left\{e_{1}, \ldots, e_{n-1}\right\}$ which is equal to $D$. Then the number of occurrences of $e_{i}$ in $e_{w}$ is half the number of intersections of $D$ with the line $x=i+1 / 2$, and the length of $e_{w}$ is $\ell(D)$.

Proof. This is a consequence of [4, Lemma 4.3.5].

\section{The MAin Results}

We can now state the two main theorems of this paper, which show how to realize the generalized Temperley-Lieb algebras of types $B$ and $D$ in terms of decorated tangles. Most of the rest of the paper will be devoted to proving these results.

We first deal with type $B$. In this case, we assume that the base ring contains $1 / 2$, so that in particular, that we are not in the situation of characteristic 2 .

Figure 8. Relations for type $B$

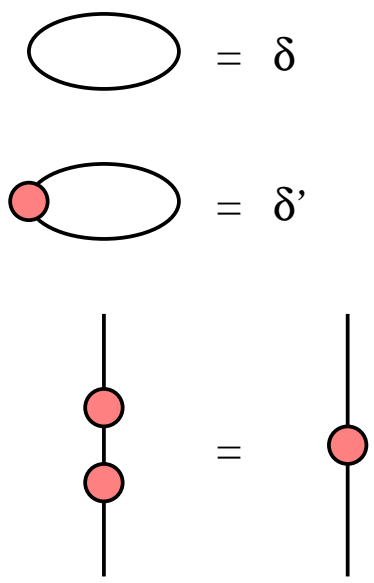

Theorem 4.1. The algebra $T L\left(B_{n}\right)$ arises from $\mathbb{D T}_{n+1}$ as an algebra of diagrams via generators $\left\{e_{\overline{1}}, e_{2}, \ldots, e_{n}\right\}$ and relations shown in Figure 8.

There is a basis for $T L\left(B_{n}\right)$ which is in natural bijection with elements of $\mathbb{D} \mathbb{T}_{n+1}$ which have no loops, at most one decoration on each edge, and which satisfy one of the following three mutually exclusive conditions:

(1) Node 1 in the north face is joined to node 1 in the south face by an undecorated edge, and there are no decorated edges.

$\left(1^{\prime}\right)$ Node 1 in the north face is joined to node 1 in the south face by an decorated edge, but there are no other decorated edges. Also, there is at least one non-propagating edge.

(2) The edges emerging from node 1 in the north face and node 1 in the south face are distinct and both decorated.

We say that an element of $\mathbb{D} \mathbb{T}_{n+1}$ which satisfies these hypotheses is $B$-admissible of type $1,1^{\prime}$ or 2 , depending on which of the three conditions above it satisfies.

This correspondence identifies $E_{1}$ with $2 e_{\overline{1}}$ and $E_{i}$ with $e_{i}$ for $i>1$.

The force of the relations in Figure 8 is firstly to exclude any edge which carries more than one decoration, and secondly to exclude any loops. The third relation 
in Figure 8 means that all edges and loops may be taken to carry $r$ decorations $(r<2)$, and the other two relations explain how to remove the loops.

A simple case by case check verifies that the $B$-admissible diagrams together with the relations in Figure 8 span an associative algebra (which we will refer to as $\left.\mathcal{T}\left(B_{n}\right)\right)$. In particular, the relations are not ambiguous.

The case of type $D$ has a similar overall feel, although there is no restriction on the characteristic of the base ring.

Figure 9. Relations for type $D$

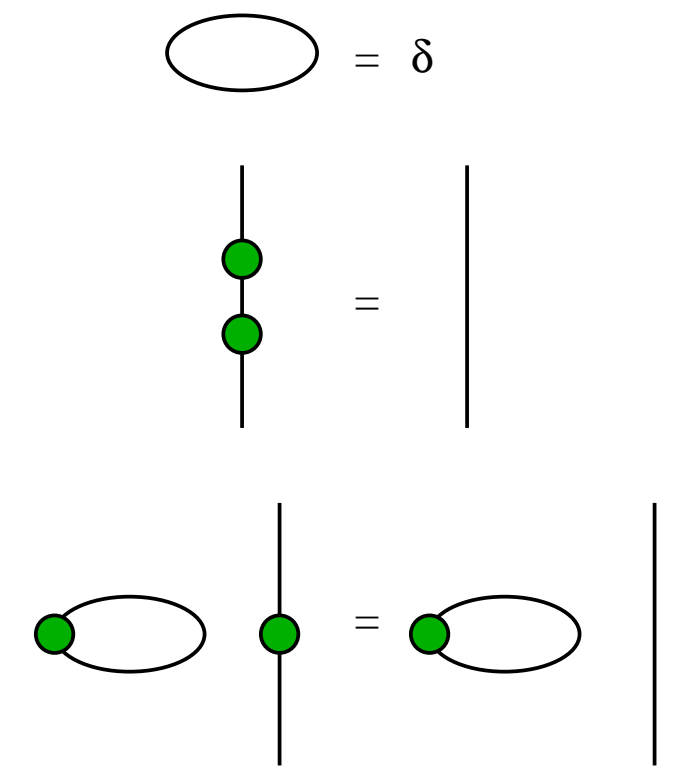

Theorem 4.2. The algebra $T L\left(D_{n}\right)$ arises from $\mathbb{D T}_{n}$ as an algebra of diagrams via generators $\left\{e_{\overline{1}}, e_{1}, e_{2}, \ldots, e_{n-1}\right\}$ and relations shown in Figure 9.

There is a basis for $T L\left(D_{n}\right)$ which is in natural bijection with elements of $\mathbb{D T}_{n}$ which have at most one decoration on each edge or loop, and which satisfy one of the following two mutually exclusive conditions:

(1) The diagram contains one loop which is decorated, and no other loops or decorations. Also, there is at least one non-propagating edge in the diagram.

(2) The diagram contains no loops and the total number of decorations is even.

We say that an element of $\mathbb{D T}_{n}$ which satisfies these hypotheses is D-admissible of type 1 or 2, depending on which of the two conditions above it satisfies.

The correspondence identifies $E_{\overline{1}}$ with $e_{\overline{1}}$ and $E_{i}$ with $e_{i}$ for all other $i$.

The third relation in Figure 9 means that any arc loses its decoration in the presence of a decorated loop. Using the first and third relations, all loops may be removed from the image of a diagram except the last decorated loop, if there is one. The second relation ensures that no arc may carry more than one decoration.

Associativity follows by considering triple products of diagrams $D_{1} D_{2} D_{3}$, first in the case where the triple product contains a loop with an odd number of decorations, and then in the other case.

It follows from these observations that the $D$-admissible diagrams of $\mathbb{D T}_{n}$ together with the relations in Figure 9 span an associative algebra (which we will refer to as $\mathcal{T}\left(D_{n}\right)$ ), under the diagram multiplication. 


\section{Combinatorics of DeCorated tangles}

In order to prove the main results of $\S 4$, we study the combinatorics of Martin and Saleur's so-called "blob algebra", which a two-parameter version of the algebra studied in [2], and is defined in terms of decorated tangles. We can associate reduced words to the blob algebra, as we did for the Temperley-Lieb algebra in $\S 3$. The proofs of theorems 4.1 and 4.2 will be tackled in $\S 6$ by considering reduced words which satisfy certain additional properties.

The blob algebra $b_{n}\left(\delta, \delta^{\prime}\right)$ is the algebra which arises from $\mathbb{D T}_{n}$ as an algebra of diagrams via generators $\left\{e, e_{1}, e_{2}, \ldots, e_{n-1}\right\}$ and the relations in Figure 8 . The parameters $\delta$ and $\delta^{\prime}$ are indeterminates.

A "blob diagram" for $b_{n}$ is an element of $\mathbb{D T}_{n}$ which has at most one decoration on each edge and no loops.

The following result was proved in $[\mathbf{1 3}]$.

Proposition 5.1 (Martin, Saleur). The associative algebra $b_{n}\left(\delta, \delta^{\prime}\right)$ has as a basis all the blob diagrams and has multiplicative structure determined by the relations in Figure 8.

Notice that the product of two blob diagrams, using the relations in Figure 8, is a scalar multiple of another one. The following lemma is easily verified.

Lemma 5.2. The following relations hold in $b_{n}\left(\delta, \delta^{\prime}\right)$ :

$$
\begin{aligned}
e_{i} e_{j} & =e_{j} e_{i} \quad \text { if }|i-j|>1 ; \\
e_{i} e_{j} e_{i} & =e_{i} \quad \text { if }|i-j|=1 ; \\
e_{i}^{2} & =\delta e_{i} ; \\
e^{2} & =e ; \\
e_{1} e e_{1} & =\delta^{\prime} e_{1} ; \\
e_{i} e & =e e_{i} \quad \text { if } i>1 .
\end{aligned}
$$

A reduced word for $b_{n}\left(\delta, \delta^{\prime}\right)$ is a monomial in the generators $\left\{e, e_{1}, e_{2}, \ldots, e_{n-1}\right\}$ of minimal length. In other words, any sequence of relations in Lemma 5.2 which can be applied to the monomial consists only of applications of the commutation relations, (1) and (6). As in the case of $T L_{n}$, it is clear that the reduced words form a spanning set for the algebra. It also follows from the results in $[\mathbf{1 3}]$ that the diagram basis for the blob algebra is the same as the one arising from the reduced words.

By using Lemma 3.2, which concerns extremal generators in reduced words for $T L_{n}$, we can show that the occurrences of $e$ and $e_{1}$ in a reduced word for $b_{n}=$ $b_{n}\left(\delta, \delta^{\prime}\right)$ alternate. (Recall that $e$ commutes with all the generators except $e_{1}$.)

Lemma 5.3. Let $e_{w}$ be a reduced word for $b_{n}$. Then there is an occurrence of $e$ between each pair of occurrences of $e_{1}$, and an occurrence of $e_{1}$ between each pair of occurrences of $e$.

Proof. Suppose there are two occurrences of $e$ in $e_{w}$. Then if there is no occurrence of $e_{1}$ between them, we can apply relation (6) until the two occurrences of $e$ are adjacent, and then apply relation (4). This is a contradiction because $e_{w}$ is reduced.

Suppose there are two occurrences of $e_{1}$ in $e_{w}$, occurring at positions $c$ and $d$, where $c<d$. If there is no occurrence of $e$ between the two occurrences of $e_{1}$, then

$$
e_{i_{c+1}} e_{i_{c+2}} \cdots e_{i_{d-1}}
$$


is a reduced word for $T L_{n}$ containing two occurrences of $e_{1}$, which contradicts Lemma 3.2. This completes the proof.

We now define two subsets of reduced words for $b_{n}$ : those satisfying the $B$ condition and those satisfying the $D$-condition. The reason for the names is of course that they will be useful in dealing with the generalized Temperley-Lieb algebras of types $B$ and $D$, respectively.

Let $e_{w}$ be a reduced word for $b_{n}$.

We say $e_{w}$ satisfies the $B$-condition if one of the following two conditions holds.

(1) Neither $e_{1}$ nor $e$ occurs in $e_{w}$.

(2) Both $e_{1}$ and $e$ occur in $e_{w}$ but there is no occurrence of $e_{1}$ to the left of the

leftmost occurrence of $e$ and there is no occurrence of $e_{1}$ to the right of the rightmost occurrence of $e$.

We say $e_{w}$ satisfies the $D$-condition if $e$ occurs in $e_{w}$ an even number of times (possibly zero).

If a reduced word $e_{w}$ satisfies the $B$-condition or the $D$-condition, the following results show that we can essentially forget about $e$ and restrict our attention to $e_{\overline{1}}=e e_{1} e$.

Lemma 5.4. Let $e_{w}$ be a reduced word satisfying the B-condition. Then $e_{w}$ is equal to a word $e_{w}^{\prime}$ in the generators

$$
\left\{e_{\overline{1}}, e_{2}, e_{3}, \ldots, e_{n-1}\right\} .
$$

Proof. We describe a procedure for constructing $e_{w}^{\prime}$.

First, for each occurrence of $e$ in $e_{w}$ which appears between two occurrences of $e_{1}$, replace $e$ by two occurrences of $e$. The $B$-condition now guarantees that each occurrence of $e_{1}$ appears between two occurrences of $e$.

Next, for each occurrence of $e_{1}$, we can commute the two surrounding occurrences of $e$ towards the occurrence of $e_{1}$ to form subsequences $e e_{1} e$. This produces a word of the desired form.

We present some examples from $b_{5}$ to illustrate the $B$-condition and Lemma 5.4.

Examples. The words $e e_{1}, e_{2} e, e_{1} e_{3}$ and $e$ do not satisfy the $B$-condition, although they are reduced.

The words $e_{3} e_{2} e_{4} e_{3}$ and $e e_{2} e_{1} e e_{3}$ are both reduced and satisfy the $B$-condition.

The word $e_{w}=e e_{1} e e_{2} e_{1} e$ satisfies the $B$-condition. Applying Lemma 5.4 to $e_{w}$ first doubles the middle $e$ to form $e e_{1} e e e_{2} e_{1} e$, and then commutes the generators to form $e e_{1} e e_{2} e e_{1} e=e_{\overline{1}} e_{2} e_{\overline{1}}=e_{w}^{\prime}$.

The $D$-condition leads to the following property.

Lemma 5.5. Let $e_{w}$ be a reduced word satisfying the D-condition. Then $e_{w}$ is equal to a (not necessarily reduced) word $e_{w}^{\prime}$ in the generators

$$
\left\{e_{\overline{1}}, e_{1}, e_{2}, e_{3}, \ldots, e_{n-1}\right\} \text {. }
$$

Proof. This is similar to the proof of Lemma 5.4 but slightly simpler.

We use Lemma 5.3 to see that the occurrences of $e_{1}$ and $e$ alternate in a reduced word $e_{w}$. The $D$-condition guarantees that there is an even number of occurrences 
of $e$. Suppose these occur at positions $i_{1}, i_{2}, \ldots, i_{2 k}$; we then pair the occurrences off by twinning the $e$ at position $i_{2 j-1}$ with that at position $i_{2 j}$. Next we commute each pair of occurrences of $e$ towards the unique $e_{1}$ which lies between them to form subexpressions of the form $e e_{1} e$. This produces a word $e_{w}^{\prime}$ of the desired form.

We give some examples to illustrate Lemma 5.5.

Examples. The words $e$ and $e_{2} e_{1} e e_{3}$ are reduced but do not satisfy the $D$-condition.

The words $e_{1} e_{3}$ and $e_{w}=e e_{1} e_{2} e e_{1}$ satisfy the $D$-condition. Applying Lemma 2.3.4 to $e_{w}$ pairs off the two occurrences of $e$ and commutes the rightmost one one place to the left to form $e e_{1} e e_{2} e_{1}=e_{\overline{1}} e_{2} e_{1}=e_{w}^{\prime}$.

The final combinatoric tool needed for the proofs of the main results is the correspondence between blob diagrams for $b_{n}$ and diagrams for $T L_{2 n}$ which satisfy a certain symmetry property. This correspondence produces tom Dieck's "symmetric bridges" $[\mathbf{2}, \S 1]$.

Consider a blob diagram $D$ for $b_{n}$. Break each decorated edge of $D$ at the decoration, and connect all the loose endpoints to the west wall in such a way that they do not intersect each other. (We will call this the asymmetric representation for $D$.) Now consider the diagram union its reflection in the west wall, which is a diagram for $b_{2 n}$ with no decorations. We will call this the symmetric representation of $D$.

It is not hard to see that this procedure in fact establishes a bijection between laterally symmetric diagrams for $T L_{2 n}$ and blob diagrams for $b_{n}$.

Example. The asymmetric representation of the diagram in Figure 10 is given in Figure 11, where the west wall is shown explicitly by the dotted line. The symmetric representation is obtained simply by considering the west wall as a mirror.

Figure 10. A blob diagram for $b_{6}$

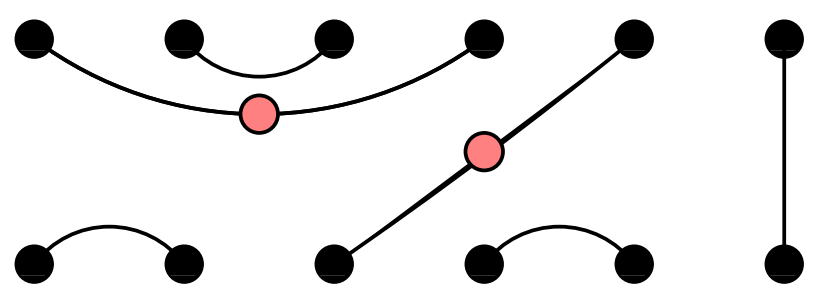

Figure 11. Asymmetric representation of Figure 10

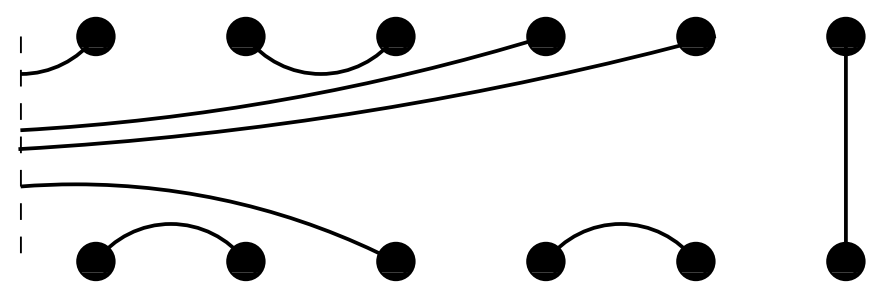

From [2, Satz 2.5], we have

Lemma 5.6. The left-right symmetric diagrams of $T L_{2 n}$ span a subalgebra of $T L_{2 n}$ of dimension $\left(\begin{array}{c}2 n \\ n\end{array}\right)$ with generators $\left\{e_{1} e_{n-1}, e_{2} e_{n-3}, \ldots, e_{2 n-1} e_{2 n+1}, e_{2 n}\right\}$.

The next result shows how the algebra of [2] is a special case of $b_{n}$. 
Lemma 5.7. If $\delta$ is invertible, the subalgebra of $T L_{2 n}\left(\delta^{2}\right)$ spanned by the left-right symmetric diagrams is isomorphic to the algebra $b_{n}(\delta, 1)$.

The isomorphism may be chosen to identify $e_{i}$ in $b_{n}$ with $e_{n+i} e_{n-i}$ in $T L_{2 n}$, and e in $b_{n}$ with $e_{n} / \delta$ in $T L_{2 n}$.

Thus $b_{n}$ has dimension

$$
\left(\begin{array}{c}
2 n \\
n
\end{array}\right) .
$$

Proof. The proof of the first two parts is simply a matter of checking that the multiplicative action of the generators is as asserted.

The third part is immediate from the correspondence between the two bases and Lemma 5.6.

It will be helpful in the proofs of the main theorems to know the significance of the total number of decorations in a blob diagram.

Lemma 5.8. Let $D$ be a blob diagram for $b_{n}$ and let $e_{w}$ be a reduced monomial corresponding to $D$. Then the number of decorations in $D$ is equal to the number of occurrences of $e$ in $e_{w}$.

Proof. Let $D^{\prime}$ be the symmetric representation of $D$, corresponding to an element of $T L_{2 n}$. We may assume that $D^{\prime}$ satisfies the hypotheses of Lemma 3.3. Lemma 3.3 shows that the number of occurrences of $e_{n}$ in a reduced monomial for $D^{\prime}$ is equal to the number of intersections with the line $x=n+1 / 2$. Lemma 5.6 shows that this is equal to the number of occurrences of $e$ in $e_{w}$. Reconstructing $D$ from $D^{\prime}$, we find that the number of decorations in $D$ is equal to the number of intersections of $D^{\prime}$ with $x=n+1 / 2$. This completes the proof.

\section{Proofs of the main Results}

We now prove Theorem 4.1. Until further notice, we replace the parameter $\delta$ by [2] and $\delta^{\prime}$ by [2]/2.

Lemma 6.1. There is a homomorphism $\rho_{B}: T L\left(B_{n}\right) \rightarrow \mathcal{T}\left(B_{n}\right)$ which takes $E_{1}$ to $2 e_{\overline{1}}$ and $E_{i}$ to $e_{i}$ for $i>1$.

Proof. This follows by checking that all the relations in Proposition 1.3 hold, which presents no difficulties.

In order to prove that $\rho_{B}$ is an isomorphism, we need to enumerate the number of $B$-admissible diagrams of the various types.

Lemma 6.2. In type $B_{n}$, the number of $B$-admissible diagrams of type 1 is $C(n)$, of type $1^{\prime}$ is $C(n)-1$ and of type 2 is $\left(\begin{array}{c}2 n \\ n\end{array}\right)-C(n)$.

This is a total of $(n+2) C(n)-1$, which is the dimension of $T L\left(B_{n}\right)$.

Proof. The diagrams of type 1 are in canonical bijection with basis diagrams for $T L_{n}$. The correspondence is given by removal of the edge joining node 1 in the north face to node 1 in the south face. The number of such diagrams is therefore equal to the Catalan number

$$
C(n):=\frac{1}{n+1}\left(\begin{array}{c}
2 n \\
n
\end{array}\right) .
$$


The case of type $1^{\prime}$ is similar. The -1 in the formula is due to the exclusion of the diagram $e$, all of whose edges are propagating (and one of which is decorated).

Let $D$ be a diagram which is either of type $1^{\prime}$ or 2 , or equal to $e$. Consider the symmetric representation of $D$ as in $\S 5$; this has the form of a basis diagram for $T L_{2 n+2}$. Observe that the diagrams which turn up in this way are precisely the symmetric diagrams in which nodes $n+1$ and $n+2$ in the north face are joined to each other, and similarly for the south face. If we remove these four nodes from the picture, as well as their associated edges, we have a bijection between the possibilities for $D$ and the set in the statement of Lemma 5.6. Thus the number of diagrams of type $1^{\prime}$ or 2 is $\left(\begin{array}{c}2 n \\ n\end{array}\right)-1$ as required: the -1 comes from the exclusion of the diagram $e$.

The assertion about the dimension of $T L\left(B_{n}\right)$ follows from $[\mathbf{3}, \S 7.2]$.

We now show that the generators given in the statement of Theorem 4.1 do indeed generate $\mathcal{T}\left(B_{n}\right)$.

Lemma 6.3. The algebra $\mathcal{T}\left(B_{n}\right)$ is generated by the set $\left\{e_{\overline{1}}, e_{2}, \ldots, e_{n}\right\}$.

Proof. Because of Lemma 5.4, we can reduce this problem to showing that any reduced $B$-admissible diagram is given by a reduced word $e_{w}$ which satisfies the $B$-condition.

It is clear that the $B$-admissible diagrams are blob diagrams and that they span a subalgebra of the blob algebra $b_{n}$. Thus, for any $B$-admissible diagram $D$, there exists a monomial $e_{w}$ in the set $\left\{e, e_{1}, e_{2}, \ldots, e_{n}\right\}$ which is equal to a scalar multiple of $D$, since every monomial is a multiple of a diagram and the monomials form a spanning set. By omitting unnecessary terms in $e_{w}$, we may assume that $e_{w}$ is reduced and that the scalar involved is 1 (i.e. $e_{w}=D$ ).

It remains to show that $e_{w}$ has the $B$-condition. If the diagram $D$ is of type 1 , this follows by Theorem 3.1 because we can choose $e_{w}$ in such a way that it avoids all occurrences of $e$ and $e_{1}$. (This uses the embedding of $T L_{n}$ in $\mathcal{T}\left(B_{n}\right)$ which sends $e_{i} \in T L_{n}$ to $e_{i+1}$.)

If $D$ is of type $1^{\prime}$ or 2 , then clearly $e D=D e=e D e=D$. Thus $e_{w}=e e_{w} e$. We now consider the relations in Lemma 5.2 which would need to be applied to the monomial $e e_{w} e$ in order to make it reduced. None of these relations alters the fact that there can be no occurrence of $e_{1}$ to the left of the leftmost $e$ or to the right of the rightmost $e$. Therefore $e_{w}$ itself has the $B$-condition if it is reduced.

The proof of Theorem 4.1 is now complete.

We can now drop the restriction that $\delta^{\prime}=\delta / 2$. Instead, we can have $\delta=v+v^{-1}$ and $\delta^{\prime}=v^{\prime}+v^{\prime-1}$, which corresponds to a quotient of a Hecke algebra $\mathcal{H}\left(B_{n}\right)$ with two independent parameters $q$ and $Q$, where $q=v^{2}$ and $Q=v^{\prime 2}$.

We now prove Theorem 4.2. First, we replace the parameter $\delta$ by [2]. This should be regarded as a change of notation rather than a restriction, because $v+v^{-1}$ may be assigned any value if $v$ takes values in an algebraically closed field.

Lemma 6.4. There is a homomorphism $\rho_{D}: T L\left(D_{n}\right) \rightarrow \mathcal{T}\left(D_{n}\right)$ which takes $E_{\overline{1}}$ to $e_{\overline{1}}$ and $E_{i}$ to $e_{i}$ for all other $i$.

Proof. This follows by checking that all the relations in Proposition 1.4 hold. The most notable relation is that $e_{1} e_{\overline{1}}=e_{\overline{1}} e_{1}$.

In order to prove that $\rho_{D}$ is an isomorphism, we need to enumerate the number of $D$-admissible diagrams of the various types. 
Lemma 6.5. In type $D_{n}$, the number of $D$-admissible diagrams of type 1 is $C(n)-$ 1 , and the number of type 2 is $\frac{1}{2}\left(\begin{array}{c}2 n \\ n\end{array}\right)$.

This is a total of

$$
\left(\frac{n+3}{2}\right) C(n)-1,
$$

which is the dimension of $T L\left(D_{n}\right)$.

Proof. The diagrams of type 1 are in canonical bijection with the nonidentity basis diagrams for $T L_{n}$ : the correspondence is given by removal of the decorated loop. The number of such diagrams is therefore $C(n)-1$.

We argue that the number of diagrams of type 2 is exactly half the number of blob diagrams for $b_{n}$, which we know to be $\left(\begin{array}{c}2 n \\ n\end{array}\right)$. Consider the permutation induced on the set of blob diagrams for $b_{n}$ by the map $\omega$ defined as follows. Let $D$ be such a diagram. We define the edge $E$ to be the one connected to the node in the northwest corner of $D$. Then $\omega(D)$ is obtained from $D$ by toggling the decoration on the edge $E$, that is, decorating $E$ if $E$ is undecorated, and removing the decoration from $E$ if $E$ is decorated. It is clear that the orbits of the action of the permutation group generated by $\omega$ are all of size 2, and that exactly one element in each orbit has an even number of decorations. Thus the number of blob diagrams with an even number of decorations is exactly half of the total, and the claim follows, completing the proof.

The assertion about the dimension of $T L\left(D_{n}\right)$ follows from $[\mathbf{3}, \S 6.2]$.

We now show that the generators given in the statement of Theorem 4.2 do indeed generate $\mathcal{T}\left(D_{n}\right)$.

Lemma 6.6. The algebra $\mathcal{T}\left(D_{n}\right)$ is generated by the set

$$
\left\{e_{\overline{1}}, e_{1}, e_{2}, \ldots, e_{n-1}\right\} .
$$

Proof. Because of Lemma 5.5, we can reduce this problem to showing that any reduced $D$-admissible diagram is given by a reduced word $e_{w}$ which satisfies the $D$-condition.

Let $D$ be a $D$-admissible diagram. If $D$ is of type 1 , then let $D_{A}$ be the diagram obtained from $D$ by removing the decorated loop. This means $D_{A}$ is a diagram for $T L_{n}$, and is equal to a monomial $e_{w}$ in the generators $\left\{e_{1}, e_{2}, \ldots, e_{n-1}\right\}$. Furthermore, $e_{w}$ is not trivial since $D$ is not allowed to be the identity diagram.

Let $a$ be minimal such that $e_{a}$ occurs in $e_{w}$. Define $e_{a}^{\prime}$ to be

$$
e_{a} e_{a-1} \cdots e_{2} e_{1} e_{\overline{1}} e_{2} \cdots e_{a-1} e_{a}
$$

or $e_{1} e_{\overline{1}}$ if $a=1$. Define $e_{w}^{\prime}$ to be the monomial in the generators for $\mathcal{T}\left(D_{n}\right)$ obtained by replacing the leftmost occurrence of $e_{a}$ in $e_{w}$ by $e_{a}^{\prime}$. Then one may easily check that $e_{w}^{\prime}$ gives the diagram $D$. (Note that we have not assumed $e_{w}^{\prime}$ is reduced.)

Now assume that $D$ is a $D$-admissible diagram of type 2 . This means that $D$ has the form of a blob diagram $D_{b}$ which is equal (by an argument like that in the proof of Lemma 6.3) to a reduced monomial $e_{w}$ in the set $\left\{e, e_{1}, e_{2}, \ldots, e_{n}\right\}$. Since $D$ has an even number of decorations, Lemma 5.8 shows that $e_{w}$ contains an even number of occurrences of $e$. This shows that $e_{w}$ has the $D$-condition, and can therefore be written as a monomial $e_{w}^{\prime}$ in the generators for $\mathcal{T}\left(D_{n}\right)$. 
Since $e_{w}$ is reduced, Lemma 5.8 shows that the number of decorations in $e_{w}$ is equal to the number of occurrences of $e$ in $e_{w}$. Therefore the third relation in Figure 8 is never needed in building up the monomial $e_{w}$. The fact that $e_{w}$ is reduced also means that the parameters $\delta$ and $\delta^{\prime}$ and their associated loops never appear. Thus the monomial for $\mathcal{T}\left(D_{n}\right)$ which has the same form as $e_{w}^{\prime}$ is equal to $D$ (since none of the diagram relations in Figure 9 are ever used in multiplying out the monomial). The proof now follows.

This completes the proof of Theorem 4.2.

\section{Applications}

We conclude by mentioning some of the applications of theorems 4.1 and 4.2. The details, which are not hard to fill in, are left to the reader.

A natural idea is to extend the algebra $T L\left(B_{n}\right)$ by adding in the generator $e$. This larger algebra can be shown to decompose into a direct sum of $T L_{n}$ and $b_{n}\left(\delta, \delta^{\prime}\right)$, which casts light on the representation theory of $T L\left(B_{n}\right)$.

Similarly, by adding the diagram $G$ (see Figure 12), $T L\left(D_{n}\right)$ may be extended to be isomorphic to a direct sum of $T L_{n}$ and an algebra $d_{n}$ which is half the dimension of $b_{n}$. The algebra $d_{n}$ can also be constructed from $T L\left(D_{n}\right)$ by treating any loop carrying an odd number of decorations as zero.

Figure 12. The diagram $G$ for $n=6$

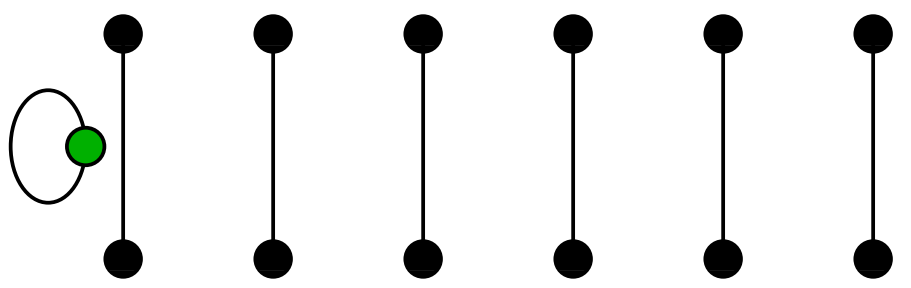

Using the results of $[\mathbf{1 3}, \S 4]$, vertex representations of these algebras may be constructed. These are representations on a tensor space $V^{\otimes n}$, where $V$ is of dimension 2; such a construction is familiar from statistical mechanics [1]. The algebra $d_{n}$ has a particularly natural realisation viewed in this way as a subalgebra of $b_{n}$.

One of the important applications of the diagram calculi is the description of the cellular structures of the algebras $T L\left(B_{n}\right)$ and $T L\left(D_{n}\right)$. This uses techniques similar to those in type $A$ : a two-sided cell (in the sense of [7]) consists of a set of diagrams with particular combinatoric properties. These properties include the number and type of propagating edges in the diagram. Viewed in this way, the diagrams can be split into a top part and a bottom part, to form parenthesis diagrams generalizing those for the Temperley-Lieb algebra [15, §2]. The parenthesis diagrams can then be used to formulate branching rules for the generically irreducible modules, thus showing for example how such modules for $T L\left(B_{n}\right)$ restrict to modules for $T L\left(B_{n-1}\right)$.

It is hoped that there will be further applications of these results to operator algebras and subfactors.

\section{ACKNOWLEDGEMENTS}

The work for this paper was done while the author was supported in part by an E.P.S.R.C. postdoctoral research assistantship. The author is grateful to the referee for pointing out some errors in an earlier version of this paper. 


\section{REFERENCES}

[1] R.J. Baxter, Exactly solved models in statistical mechanics, Academic Press, New York, 1982.

[2] T. tom Dieck, Symmetrische Brücken und Knotentheorie zu den Dynkin-Diagrammen vom Typ B, J. reine angew. Math. 451 (1994), 71-88.

[3] C.K. Fan, Structure of a Hecke Algebra Quotient, Jour. Amer. Math. Soc. 10 (1997), 139-167.

[4] C.K. Fan and R.M. Green, On the affine Temperley-Lieb algebras, Jour. L.M.S. (to appear).

[5] P.J. Freyd and D.N. Yetter, Braided compact closed categories with applications to low dimensional topology, Adv. Math. 77 (1989), 156-182.

[6] J.J. Graham, Modular representations of Hecke algebras and related algebras, Ph.D. thesis, University of Sydney, 1995.

[7] J.J. Graham and G.I. Lehrer, Cellular Algebras, Invent. Math. 123 (1996), 1-34.

[8] R.M. Green, Cellular algebras arising from Hecke algebras of type $H_{n}$, Math. Zeit. (to appear).

[9] J.E. Humphreys, Reflection Groups and Coxeter Groups, Cambridge University Press, Cambridge, 1990.

[10] V.F.R. Jones, Hecke algebra representations of braid groups and link polynomials, Ann. of Math. (2) 126 (1987), 335-388.

[11] L.H. Kauffman, State models and the Jones polynomial, Topology 26 (1987), 395-407.

[12] An invariant of regular isotopy, Trans. Amer. Math. Soc. 318 (1990), 417-471.

[13] P. Martin and H. Saleur, The blob algebra and the periodic Temperley-Lieb algebra, Lett. Math. Phys. 30 (3) (1994), 189-206.

[14] H.N.V. Temperley and E.H. Lieb, Relations between percolation and colouring problems and other graph theoretical problems associated with regular planar lattices: some exact results for the percolation problem, Proc. Roy. Soc. London Ser. A 322 (1971), 251-280.

[15] B.W. Westbury, The representation theory of the Temperley-Lieb Algebras, Math. Zeit. 219 (1995), 539-565. 